MATHEMATICS OF COMPUTATION

Volume 67, Number 223, July 1998, Pages 987-999

S $0025-5718(98) 00956-9$

\title{
INTERPOLATION CORRECTION FOR COLLOCATION SOLUTIONS OF FREDHOLM INTEGRO-DIFFERENTIAL EQUATIONS
}

\author{
QIYA HU
}

\begin{abstract}
In this paper we discuss the collocation method for a large class of Fredholm linear integro-differential equations. It will be shown that, when a certain higher order interpolation operation is added to the collocation solution of this equation, the new approximations will, under suitable assumptions, admit a multiterm error expansion in even powers of the step-size $h$. Based on this expansion, ideal multilevel correction results of this collocation solution are obtained.
\end{abstract}

\section{INTRODUCTION}

We consider the integro-differential boundary value problem

$$
\left\{\begin{array}{l}
\sum_{i=0}^{m} a_{i}(t) D^{i} u(t)-\int_{0}^{1} \sum_{i=0}^{m} k_{i}(t, s) D^{i} u(s) d s=f(t), \quad t \in J=[0,1], \\
\sum_{i=0}^{m-1}\left[\gamma_{j, i} D^{i} u(0)+\gamma_{j, m+i} D^{i} u(1)\right]=0, \quad j=1, \ldots, m
\end{array}\right.
$$

where $m$ is a natural number; the function $a_{m}$ possesses no zeros and hence may be assumed without loss of generality to be identically $1 ;\left(\gamma_{j, i}\right)$ is a real $(m, 2 m)$ matrix. It will always be assumed that (1.1) possesses a unique solution $u \in C^{m}(J)$.

Equation (1.1) encompasses some important particular cases frequently encountered in physical modelling processes, and there is some literature on its numerical solution ([2]-[4], [6]-[8]). For example, Volk [8] discussed the superconvergence of the iterated Galerkin approximation to equation (1.1); the author [4] discussed the extrapolation for the iterated Galerkin approximation to a particular case of (1.1) (i.e., $a_{i}=0$ for $0 \leq i \leq m-1$ ).

In the present paper we give a complete analysis of a multilevel correction method for the collocation solution of (1.1). This correction method depends on a certain higher order interpolation procedure instead of the Sloan iteration, and has obvious advantages over the traditional extrapolation method (see Section 2). The results obtained in this paper compare favourably with the corresponding results for iterated Galerkin solutions of Fredholm linear integral equations of the second kind (compare [10]). The numerical results given in Section 5 will confirm this inference further.

Received by the editor January 10, 1995 and, in revised form, August 9, 1995 and October 22, 1996.

1991 Mathematics Subject Classification. Primary 65B10, 45D05, 65R20.

This work was partially supported by the National Science Foundation.

(C)1998 American Mathematical Society 


\section{MAin Results}

It is necessary to write (1.1) in an operator form.

The operators $K: C^{m}(J) \rightarrow C(J), L: C^{m}(J) \cap R^{-1}\{0\} \rightarrow C(J)$ and $L^{*}$ : $C^{m-1}(J) \cap R^{-1}\{0\} \rightarrow C(J)$ are, respectively, defined by

$$
\begin{gathered}
K g(t)=\int_{0}^{1} \sum_{i=0}^{m} k_{i}(t, s) D^{i} g(s) d s, \quad t \in J, \\
L g(t)=\sum_{i=0}^{m} a_{i}(t) D^{i} g(t), \quad L^{*} g(t)=-\sum_{i=0}^{m-1} a_{i}(t) D^{i} g(t), \quad t \in J,
\end{gathered}
$$

where $R^{-1}\{0\}$ describes the nullspace of the operator

$$
\begin{gathered}
R: C^{m-1}(J) \rightarrow \mathbf{R}^{m}, \\
g \rightarrow\left(\sum_{i=0}^{m-1}\left[\gamma_{j, i} D^{i} g(0)+\gamma_{j, m+i} D^{i} g(1)\right]\right)_{j=1}^{m} .
\end{gathered}
$$

Set $L_{1}=L+L^{*}\left(=D^{m}\right)$ and $K_{1}=K+L^{*}$. Equation (1.1) can be written as

$$
\left(L_{1}-K_{1}\right) u(t)=f(t), \quad t \in J,
$$

where the restriction of $K_{1}$ to the domain of $K_{1}$ is also denoted by the symbol $K_{1}$.

We assume that $L$ and $L_{1}-K_{1}$ (i.e. $L-K$ ) are continuously invertible, and $K$ is compact with respect to the norms

$$
\|\bullet\|_{W_{\infty}^{m}}:=\left(g \rightarrow \max _{0 \leq i \leq m}\left\{\left\|D^{i} g\right\|_{\infty}\right\}\right)
$$

(these hypotheses are standard, refer to [8]). The space $W_{\infty}^{m}(J) \cap R^{-1}\{0\}$ is abbreviated to $W_{\infty, R}^{m}$.

For a given integer $N \geq 1$, introduce the mesh points $t_{n}=n h, n=0, \ldots, N$, with $h=1 / N$. Set $e_{n}=\left[t_{n-1}, t_{n}\right](n=1, \ldots, N)$. In the following we shall be concerned with the finite-dimensional spaces

$$
\bar{S}_{k-1, h}^{(-1)}:=\left\{v:\left.v\right|_{e_{n}} \in P_{k}(n=1 \text { and } n=N) \text { or }\left.v\right|_{e_{n}} \in P_{k-1}(2 \leq n \leq N-1)\right\}
$$

and

$$
S_{k, h}^{(0)}:=\left\{v: v \in C(J),\left.v\right|_{e_{n}} \in P_{k} \quad(n=1, \ldots, N)\right\} .
$$

We are looking for $u_{h} \in W_{\infty, R}^{m}$ satisfying $L_{1} u_{h} \in \bar{S}_{k-1, h}^{(-1)}\left(\right.$ or $\left.L_{1} u_{h} \in S_{k, h}^{(0)}\right)$ and

$$
\left(L_{1}-K_{1}\right) u_{h}(t)=f(t), \quad t \in \bigcup_{n=1}^{N} X_{n},
$$

where

$$
\begin{array}{r}
X_{n}:=\left\{t_{n j}: t_{n j}=\left(n-1+c_{j}\right) h, 0=c_{1}<c_{2}<\cdots<c_{k}<c_{k+1}=1\right\} \\
(n=1 \text { and } n=N)
\end{array}
$$

and

$$
X_{n}:=\left\{t_{n j}: t_{n j}=\left(n-1+c_{j}^{\prime}\right) h, 0 \leq c_{1}^{\prime}<\cdots<c_{k}^{\prime} \leq 1\right\}(2 \leq n \leq N-1),
$$

or

$X_{n}:=\left\{t_{n j}: t_{n j}=\left(n-1+c_{j}\right) h, 0=c_{1}<c_{2}<\cdots<c_{k}<c_{k+1}=1\right\}(1 \leq n \leq N)$. 
The collocation equation (2.2), together with the boundary condition $R u_{h}=$ 0 , will define a unique approximation $u_{h} \in L_{1}^{-1}\left\{\bar{S}_{k-1, h}^{(-1)}\right\}$ (or $u_{h} \in L_{1}^{-1}\left\{S_{k, h}^{(0)}\right\}$ ) whenever the step-size $h$ is sufficiently small.

Let $\pi_{h}$ denote interpolation onto $\bar{S}_{k-1, h}^{(-1)}$ (or $\left.S_{k, h}^{(0)}\right)$ at the collocation points $\left\{t_{n j}\right\}$. Then the collocation equation (2.2) can be written as

$$
\left(L_{1}-\pi_{h} K_{1}\right) u_{h}(t)=\pi_{h} f(t), \quad t \in J
$$

(note that $L_{1} u_{h} \in \bar{S}_{k-1, h}^{(-1)}$ or $L_{1} u_{h} \in S_{k, h}^{(0)}$ ).

Remark 2.1. The boundary value problem (1.1) may be written directly in the form

$$
(L-K) u(t)=f(t), \quad t \in J,
$$

thus the corresponding collocation approximation $\bar{u}_{h}$ is determined by $L \bar{u}_{h} \in \bar{S}_{k-1, h}^{(-1)}$ (or $L \bar{u}_{h} \in S_{k, h}^{(0)}$ ) and

$$
\left(L-\pi_{h} K\right) \bar{u}_{h}(t)=\pi_{h} f(t), \quad t \in J .
$$

But, when $\left\{a_{i} \mid i=0(1) m-1\right\}$ do not all vanish, the calculation of $\bar{u}_{h}$ will be difficult (refer to [8]).

The iterated Galerkin method for $(2.1)$ has been discussed in $[4,8]$. If $\widetilde{u}_{h} \in$ $L_{1}^{-1}\left\{S_{k, h}^{(0)}\right\}$ and $\widetilde{\widetilde{u}}_{h} \in L_{1}^{-1}\left\{S_{k-1, h}^{(-1)}\right\}$ denote the Galerkin approximations to (2.1), then the corresponding iterated Galerkin approximations defined by

$$
\widetilde{u}_{h}^{*}=L_{1}^{-1}\left(f+K_{1} \widetilde{u}_{h}\right), \quad \widetilde{\widetilde{u}}_{h}^{*}=L_{1}^{-1}\left(f+K_{1} \widetilde{\widetilde{u}}_{h}\right),
$$

where

$$
S_{k-1, h}^{(-1)}:=\left\{v:\left.v\right|_{e_{n}} \in P_{k-1}, n=1, \cdots, N\right\} .
$$

(i) Assume that $f, a_{i} \in C^{2 k}(J)$ and $k_{i} \in C^{2 k}(J \times J)$. Then (see [8])

$$
\left\|\widetilde{u}_{h}^{*}-u\right\|_{w_{\infty}^{m}} \leq C h^{2 k}
$$

where $C$ denotes a constant independent of $h$;

(ii) Assume that $a_{i} \equiv 0(0 \leq i \leq m-1)$. If $f \in C^{2 p+2}(J)$ and $k_{i} \in C^{2 p+2}(J \times J)$, then (see [4])

$$
D^{r} \widetilde{\widetilde{u}}_{h}^{*}=D^{r} u(t)+\sum_{i=k}^{p} \widetilde{C}_{r, i}(u, t)+\widetilde{R}_{r, h}(t), \quad t \in J, 0 \leq r \leq m,
$$

where $0 \leq \alpha \leq m ; \widetilde{C}_{r, i}(u, t)$ are independent of $h$, and $\widetilde{C}_{r, i}(u, \bullet) \in C^{2 p+2}(J)$; $\widetilde{R}_{r, h} \in C(J)$, and satisfy $\left\|\widetilde{R}_{r, h}\right\| \leq C h^{2 p+2}$. Thus the extrapolation to $D^{r} \widetilde{\widetilde{u}}_{h}^{*}$ can be done repeatedly.

By the way, the above results are also true under the corresponding Sobolev smoothness assumptions like the case of Fredholm integral equations (see [6]). This is an advantage of Galerkin method over collocation method (compare Theorem 1).

If we set $u_{h}^{*}=L_{1}^{-1}\left(f+K_{1} u_{h}\right)$, then we can show, under the usual smoothness assumptions, that $u_{h}^{*}$ possesses the same accelerated convergence properties as $\widetilde{u}_{h}^{*}$ and $\widetilde{u}_{h}^{*}$, provided that the collocation parameters $\left\{c_{j}^{\prime}\right\}$ (or $\left\{c_{j}\right\}$ ) are chosen as the $k$ Gauss points for $(0,1)$ (or the $k+1$ Lobatto points for $[0,1]$, i.e., the zeros of the $k+1$ degree polynomial $\left.Q_{k+1}(s)=\frac{2^{k}}{k !} \frac{d^{k-1}}{d s^{k-1}}[s(s-1)]^{k}\right)$. But, for numerical purposes (refer to Remark 2.2) we introduce new kinds of accelerated convergence methods for $u_{h}$, instead of the Sloan iteration mentioned above. 
For a natural number $p \geq k$, set $N^{\prime}=\left[\frac{k N}{2 p+1}\right]$. Let $J$ be divided into $N^{\prime}$ subintervals $\left\{\sigma_{r}\right\}$ such that $\sigma_{r}\left(r=1, \ldots, N^{\prime}-1\right)$ contains $2 p+2$ points of $\left\{t_{n}\right\}$ (i.e. $\left.\sigma_{r}=\left[t_{(2 p+1)(r-1)}, t_{(2 p+1) r}\right]\right)$, and $\sigma_{N^{\prime}}$ contains $q$ points of $\left\{t_{n}\right\}(q=k N+1-$ $\left.\left(N^{\prime}-1\right)(2 p+1) \geq 2 p+2\right)$. Set

$$
S(p, N):=\left\{v: v \in C(J),\left.v\right|_{\sigma_{r}} \in P_{2 p+1}\left(r=1, \ldots, N^{\prime}-1\right) \text { and }\left.v\right|_{\sigma_{N^{\prime \prime}}} \in P_{q-1}\right\},
$$

let $\bar{\pi}_{h}$ denote interpolation onto $S(p, N)$ at the points $\left\{t_{n}\right\}$. Besides, set $N^{\prime \prime}=$ $\left[\frac{k N+1}{2 p+1}\right]$, and let $J$ be divided into $N^{\prime \prime}$ subintervals $\left\{\bar{\sigma}_{i} \mid i=1(1) N^{\prime \prime}\right\}\left(\bar{\sigma}_{i}\right.$ is denoted by $\left.\left[s_{i-1}, s_{i}\right]\right)$ such that $\bar{\sigma}_{i}\left(i=1, \ldots, N^{\prime \prime}-1\right)$ contains $2 p+2$ points of $\left\{t_{n j}\right\}$, and $\bar{\sigma}_{N^{\prime \prime}}$ contains $q^{\prime}$ points of $\left\{t_{n j}\right\}\left(q^{\prime}=k N+2-\left(N^{\prime \prime}-1\right)(2 p+1) \geq 2 p+2\right)$, where $s_{0}=t_{0} ; s_{i}\left(i=1, \ldots, N^{\prime \prime}-1\right)$ is chosen as one of the collocation points $\left\{t_{n j}\right\} ; s_{N^{\prime \prime}}=t_{N}$. Set

$$
\bar{S}(p, N):=\left\{v: v \in C(J),\left.v\right|_{\bar{\sigma}_{i}} \in P_{2 p+1}\left(i=1, \ldots, N^{\prime \prime}-1\right) \text { and }\left.v\right|_{\bar{\sigma}_{N^{\prime \prime}}} \in P_{q^{\prime}-1}\right\},
$$

and let $\widetilde{\pi}_{h}$ denote interpolation onto $\bar{S}(p, N)$ at the points $\left\{t_{n j}\right\}$.

In the following discussions, $u_{h}$ denotes the collocation approximations defined by (2.2); the collocation parameters $\left\{c_{j}\right\}\left(\left\{c_{j}^{\prime}\right\}\right)$ are given by the $k+1$ Lobatto points for $[0,1]$ ( $k$ Gauss points for $(0,1))$.

Theorem 1. Let the functions $f, a_{i}$ and $k_{i}$ in (1.1) satisfy $f, a_{i} \in C^{2 p+2}(J), k_{i} \in$ $C^{2 p+2}(J \times J)$.

(i) Assume that $\left\{a_{i} \mid i=0(1) m-1\right\}$ do not all vanish. Let $u_{h} \in L_{1}^{-1}\left(S_{k, h}^{(0)}\right)$. Then

$$
\bar{\pi}_{h} D^{r} u_{h}(t)=D^{r} u(t)+\sum_{i=k}^{p} C_{r, i}(t) h^{2 i}+R_{r, h}(t), \quad t \in J, 0 \leq r \leq m,
$$

where all $C_{r, i}(t)$ are independent of $h$, and $C_{r, i} \in C^{2 p+2-2 i}(J) ; R_{r, h}(t)$ satisfy $\left\|R_{r, h}\right\|_{\infty} \leq C h^{2 p+2}$.

(ii) If $a_{i} \equiv 0(i=0, \ldots, m-1)$, and $u_{h} \in L_{1}^{-1}\left(\bar{S}_{k-1, h}^{(-1)}\right)$, then

$$
\bar{\pi}_{h} D^{r} u_{h}(t)=D^{r} u(t)+\sum_{i=k}^{p} C_{r, i}(t) h^{2 i}+R_{r, h}(t), \quad t \in J, 0 \leq r \leq m-1,
$$

and

$$
\tilde{\pi}_{h} D^{m} u_{h}(t)=D^{m} u(t)+\sum_{i=k}^{p} C_{m, i}(t) h^{2 i}+R_{m, h}(t), \quad t \in J .
$$

Remark 2.2. Theorem 1 indicates that the higher order interpolation for $D^{r} u_{h}$ possesses the same convergence behaviours and "acceleration effect" as the iterated collocation (or Galerkin) approximation for (1.1). The advantage of our method is that computing the higher order interpolation for $D^{r} u_{h}$ is cheaper than to compute the corresponding iterated collocation (or Galerkin) approximation, because for the computation of this "iterated approximation" double integrals containing the Green's function of $L_{1}$ need to be calculated. By the way, this theorem implies that the collocation approximation itself admits a fine error expansion at the knots. Thus, if the approximate solution of (1.1) is evaluated only for some mesh points, then neither the Sloan iteration nor the higher order interpolation operation need to be used. 
Now we introduce a multilevel correction method.

The sequence of collocation operators $Q_{h}: C^{m}(J) \rightarrow L_{1}^{-1}\left(\bar{S}_{k-1, h}^{(-1)}\right)\left(\right.$ or $\left.L_{1}^{-1}\left(S_{k, h}^{(0)}\right)\right)$ is defined as follows: for $v \in C^{m}(J), Q_{h} v$ is the unique solution of equation ( $h$ is sufficiently small)

$$
\left(L_{1}-\pi_{h} K_{1}\right) Q_{h} v=\pi_{h} f_{v},
$$

where $f_{v}=\left(L_{1}-K_{1}\right) v$.

Theorem 2. Let the functions $f, a_{i}$ and $k_{i}$ in (1.1) satisfy $f, a_{i} \in C^{2 k(r+1)}(J), k_{i} \in$ $C^{2 k(r+1)}(J \times J)$, where $r \in \mathbf{N}$.

(i) Assume that $\left\{a_{i} \mid i=0(1) m-1\right\}$ do not all vanish. Let $u_{h} \in L_{1}^{-1}\left(S_{k, h}^{(0)}\right)$. Then we have the multilevel correction estimates

$$
\left\|u_{h, r}-u\right\|_{W_{\infty, R}^{m}} \leq C h^{2 k(r+1)}
$$

where $u_{h, r}=(-1)^{r} \sum_{j=0}^{r}(-1)^{j} C_{r+1}^{j}\left(L_{1}^{-1} \bar{\pi}_{h} D^{m} Q_{h}\right)^{r-j} L_{1}^{-1} \bar{\pi}_{h} D^{m} u_{h}$, with $p=$ $k(r+1)-1$.

(ii) If $a_{i} \equiv 0(i=0, \ldots, m-1)$, and $u_{h} \in L_{1}^{-1}\left(\bar{S}_{k-1, h}^{(-1)}\right)$, then

$$
\left\|\bar{u}_{h, r}-u\right\|_{W_{\infty, R}^{m}} \leq C h^{2 k(r+1)},
$$

where $\bar{u}_{h, r}=(-1)^{r} \sum_{j=0}^{r}(-1)^{j} C_{r+1}^{j}\left(L_{1}^{-1} \widetilde{\pi}_{h} D^{m} Q_{h}\right)^{r-j} L_{1}^{-1} \widetilde{\pi}_{h} D^{m} u_{h}$, with $p=$ $k(r+1)-1$. (1.1).

The approximations $u_{h, r}$ and $\bar{u}_{h, r}$ are called the $r$ th-level corrected solutions of

Remark 2.3. Since the Green's function of the differential operator $L_{1}$ is a piecewise polynomial, the $r$ th-level corrected approximations $u_{h, r}$ and $\bar{u}_{h, r}$ can be computed analytically. In most applications, $k$ may be chosen as $k=1$ or $k=2$. When $k=1$, the global convergence order of $u_{h, 2}$ and $\bar{u}_{h, 2}$ will be 6 ; when $k=2$, the global convergence order of $u_{h, 1}$ and $\bar{u}_{h, 1}$ will be 8 .

Remark 2.4. Using Theorem 1 we can also obtain multilevel extrapolation results (refer to [3]). But the calculations of the $r$ th-level extrapolated approximation are heavier than ones of the $r$ th-level corrected approximation, because we have to increase the number of the knots as many as two times whenever we apply the extrapolation procedure; moreover, the global accuracy of this rth-level extrapolated approximation is only $O\left(h^{2 k+2 r}\right)$, which is much lower than that of the $r$ th-level corrected approximation unless $k=1$ (compare (2.7) or (2.8)).

Remark 2.5. In particular, Theorem 2 is true for the case of differential boundary value problems (i.e. $k_{i} \equiv 0, i=0, \ldots, m$ ). It extends the superconvergence results obtained by de Boor [1]. This is also an advantage of the collocation method over the Galerkin method, since there isn't a multilevel extrapolation (or correction) estimate of the Galerkin approximation to two-point boundary value problems. 


\section{LEMMAS}

Lemma 1. Let $e:=u_{h}-u$ denote the error function. Then e satisfies the identity relation

$$
e=\sum_{i=0}^{\mu}\left(K_{h} L^{*}\right)^{i} K_{h} L_{1} u+\left(K_{h} L^{*}\right)^{\mu+1} e
$$

where

$$
\begin{aligned}
K_{h}= & L^{-1} \sum_{j=1}^{\lambda} M_{h}^{j}+L^{-1}\left(\pi_{h}-I\right)\left(I+\sum_{j=1}^{\lambda} M_{h}^{j}\right) \\
& +L^{-1} \pi_{h}\left(I-K L_{1} \pi_{h}\right)^{-1} K L^{-1}\left(\pi_{h}-I\right) M_{h}^{\lambda},
\end{aligned}
$$

with $M_{h}=\left(I-K L^{-1}\right)^{-1} K L^{-1}\left(\pi_{h}-I\right) ; \lambda$ and $\mu$ are natural numbers (to be determined).

Proof. Subtraction of (2.1) from (2.3) leads to

$$
L_{1} e=\pi_{h} K_{1} e+\left(\pi_{h}-I\right)\left(K_{1} u+f\right) .
$$

Noting that $K_{1} u+f=L_{1} u, L_{1}=L+L^{*}$ and $K_{1}=K+K^{*}$, (3.9) may be written in the form

$$
e=L^{-1} \pi_{h} K e+L^{-1}\left(\pi_{h}-I\right)\left(L_{1} u+L^{*} e\right) .
$$

On the other hand, from (3.3) we have (since $I-K L^{-1} \pi_{h}$ has continuous inverse for sufficiently small $h$ )

$$
K e=\left(I-K L_{1} \pi_{h}\right)^{-1} K L^{-1}\left(\pi_{h}-I\right)\left(L_{1} u+L^{*} e\right) .
$$

Thus, if we substitute (3.4) into (3.3), then we obtain

$$
\begin{aligned}
e= & L^{-1} \pi_{h}\left(I-K L_{1} \pi_{h}\right)^{-1} K L^{-1}\left(\pi_{h}-I\right)\left(L_{1} u+L^{*} e\right) \\
& +L^{-1}\left(\pi_{h}-I\right)\left(L_{1} u+L^{*} e\right) .
\end{aligned}
$$

Using the following identity relation repeatedly

$\left(I-K L_{1} \pi_{h}\right)^{-1} K L^{-1}=\left(I-K L^{-1}\right)^{-1}+\left(I-K L_{1} \pi_{h}\right)^{-1} K L^{-1}\left(\pi_{h}-I\right)\left(I-K L^{-1}\right)^{-1}$, expression (3.5) yields that

$$
\begin{aligned}
e= & L^{-1} \pi_{h}\left[\sum_{j=1}^{\lambda} M_{h}^{j}+\left(I-K L_{1} \pi_{h}\right)^{-1} K L^{-1}\left(\pi_{h}-I\right) M_{h}^{\lambda}\right]\left(L_{1} u+L^{*} e\right) \\
& +L^{-1}\left(\pi_{h}-I\right)\left(L_{1} u+L^{*} e\right)
\end{aligned}
$$

namely

$$
e=K_{h} L_{1} u+K_{h} L^{*} e .
$$

Furthermore, from (3.6) we have

$$
e=K_{h} L_{1} u+K_{h} L^{*} K_{h} L_{1} u+\left(K_{h} L^{*}\right) e .
$$

Successively, we can deduce (3.1).

The following result is standard (refer to [4], [8]).

Lemma 2. There exists a positive number $\varepsilon$ such that

$$
\left\|\left(I-K L^{-1} \pi_{h}\right)^{-1}\right\|_{C(J) \rightarrow C(J)} \leq C, \quad h<\varepsilon .
$$


The following lemma can be proved as in [5] Lemma 5 (note that $Q_{k+1}^{\prime}(s)$ is just the shifted Legendre polynomial $\left.L_{k}(2 s-1)\right)$.

Lemma 3. Let $q \geq m$. If $\varphi \in C^{2 q+2}(J), \psi \in C^{2 q+2-k}(J)$, then the following expansions are valid for all $e_{n}$

$$
\int_{e_{n}}\left(\pi_{h}-I\right) \varphi \bullet \psi d t=\sum_{j=k}^{q} h^{2 j} \sum_{i=k}^{2 j} C_{i j} \int_{e_{n}} D^{2 j-i}\left(D^{i} \varphi \bullet \psi\right) d t+O\left(h^{2 q+3}\right),
$$

$$
\begin{aligned}
\int_{e_{n}} D^{\alpha}\left(\pi_{h}-I\right) \varphi \bullet \psi d t & \\
= & \sum_{r=1} C_{\alpha}^{r} \sum_{j=\alpha_{1}}^{\alpha_{2}} h^{2 j} \sum_{i=k}^{2 j+r} C_{i j r} \int_{e_{n}} D^{2 j+r-i}\left(D^{i+\alpha-r} \varphi \bullet \psi\right) d t \\
& +\sum_{j=k}^{\alpha_{2}} h^{2 j} \sum_{i=k}^{2 j} C_{i j} \int_{e_{n}} D^{2 j-i}\left(D^{i+\alpha} \varphi \bullet \psi\right) d t+O\left(h^{2 \alpha_{2}+3}\right),
\end{aligned}
$$

where $1 \leq \alpha \leq k, \alpha_{1}=\left[\frac{i-r+1}{2}\right], \alpha_{2}=\left[q-\frac{\alpha}{2}\right] ; C_{i j}, C_{i j r}$ are constants independent of $h$.

Now, we introduce a new concept.

A sequence of functions $G_{h}$ is said to be "expansible" if there are functions $G_{j i}$ and $\bar{G}_{r j i}$ independent of $h$ such that the following integral expansions are valid for all $e_{n}$ and $k \in C^{2 q+2-k}(J)$ :

$$
\begin{array}{r}
\int_{e_{n}} k(t) G_{h}(t) d t=\sum_{j=k}^{q} h^{2 j} \sum_{i=0}^{2 j-k} \int_{e_{n}} G_{j i}(t) \bullet D^{i} k(t) d t+O\left(h^{2 q+3}\right), \\
\int_{e_{n}} k(t) \bullet D^{r} G_{h}(t) d t=\sum_{j=r_{1}}^{r_{2}} h^{2 j} \sum_{i=0}^{r_{3}} \int_{e_{n}} \bar{G}_{r j i}(t) \bullet D^{i} k(t) d t+O\left(h^{2 r_{2}+3}\right) .
\end{array}
$$

Here $k \leq q \leq p, G_{j i} \in C^{2 q+2-2 j}(J), \bar{G}_{r j i} \in C^{2 q+2-2 j-r}(J) ; 1 \leq r \leq 2 q, r_{1}=$ $\max \left\{0,\left[\frac{k+1-r}{2}\right]\right\}, r_{2}=\left[q-\frac{r}{2}\right], r_{3}=2 j+r-k$.

Set $\Omega_{1}:=\{(t, s): 0 \leq s \leq t \leq 1\}$ and $\Omega_{2}:=\{(t, s): 0 \leq t \leq s \leq 1\}$.

Lemma 4. Assume that $G_{h}$ is "expansible". Then $\left(\pi_{h}-I\right) G_{h}$ is also "expansible", $\int_{0}^{t} R_{1}(t, s) G_{h}(s) d s$ is "expansible" for $R_{1} \in C^{2 p+2}\left(\Omega_{1}\right)$ and $\int_{t}^{1} R_{2}(t, s) G_{h}(s) d s$ is "expansible" for $R_{2} \in C^{2 p+2}\left(\Omega_{2}\right)$.

Proof. It is obvious that $\left(\pi_{h}-I\right) G_{h}$ is "expansible" (using Lemma 3 and (3.11)). 
Let $k \in C^{2 q+2-k}(J)$. By (3.10), there are functions $G_{j i}$ independent of $h$ such that

$$
\begin{aligned}
\int_{e_{n}}[ & \left.k(t) \int_{0}^{t} R_{1}(t, s) G_{h}(s) d s\right] d t \\
= & \int_{e_{n}}\left[k(t) \int_{0}^{t_{n-1}} R_{1}(t, s) G_{h}(s) d s\right] d t \\
& +\int_{e_{n}}\left[k(t) \int_{t_{n-1}}^{t} R_{1}(t, s) G_{h}(s) d s\right] d t \\
= & \sum_{j=k}^{q} h^{2 j} \sum_{i=0}^{2 j-k} \int_{e_{n}}\left[k(t) \int_{0}^{t_{n-1}} G_{j i}(s) \partial_{s}^{i} R_{1}(t, s) d s\right] d t+I_{n}(t),
\end{aligned}
$$

where $G_{j i} \in C^{2 q+2-2 j}(J)$.

On the other hand, changing the order of integration and using (3.10), we obtain

$$
\begin{aligned}
I_{n}(t)= & \int_{e_{n}}\left[\int_{s}^{t_{n}} k(t) R_{1}(t, s) d t \bullet G_{h}(s)\right] d s \\
= & \sum_{j=k}^{q} h^{2 j} \sum_{i=0}^{2 j-k} \int_{e_{n}}\left[G_{j i}(s) \partial_{s}^{i} \int_{s}^{t_{n}} k(t) R_{1}(t, s) d t\right] d s \\
= & \sum_{j=k}^{q} h^{2 j} \sum_{i=0}^{2 j-k} \int_{e_{n}}\left[G_{j i}(s) \int_{s}^{t_{n}} \partial_{s}^{i} R_{1}(t, s) k(t) d t\right] d s \\
& +\sum_{j=k}^{q} h^{2 j} \sum_{i=0}^{2 j-k-1} \int_{e_{n}} \bar{G}_{j i}(s) D^{i} k(s) d s,
\end{aligned}
$$

where $\bar{G}_{j i} \in C^{2 q+2-2 j}(J)$.

Changing the order of integration once again yields that

$$
\int_{e_{n}}\left[G_{j i}(s) \int_{s}^{t_{n}} \partial_{s}^{i} R_{1}(t, s) k(t) d t\right] d s=\int_{e_{n}}\left[k(t) \int_{t_{n-1}}^{t} G_{j i}(s) \partial_{s}^{i} R_{1}(t, s) d s\right] d t .
$$

From (3.12), (3.13) and (3.14), we know that $\int_{0}^{t} R_{1}(t, s) G_{h}(s) d s$ satisfies (3.10). Analogously, we can show that $\int_{0}^{t} R_{1}(t, s) G_{h}(s) d s$ satisfies (3.11), and $\int_{t}^{1} R_{2}(t, s) G_{h}(s) d s$ satisfies (3.10), (3.11). Thus, both $\int_{0}^{t} R_{1}(t, s) G_{h}(s) d s$ and $\int_{t}^{1} R_{2}(t, s) G_{h}(s) d s$ are "expansible".

Lemma 5 ([6]). If $f, a_{i} \in C^{2 p+2}(J)$ and $k_{i} \in C^{2 p+2}(J \times J)$, then $u \in C^{2 p+2+m}(J)$.

Set

$$
\begin{gathered}
A_{h}=L^{-1}\left(\sum_{j=1}^{\lambda} M_{h}^{j}\right), \quad B_{h}=L^{-1}\left(\pi_{h}-I\right)\left(I+\sum_{j=1}^{\lambda} M_{h}^{j}\right), \\
C_{r, h}=\left(A_{h} L^{*}+B_{h} L^{*}\right)^{r-1}\left(A_{h}+B_{h}\right) .
\end{gathered}
$$


Lemma 6. Assume that the smoothness assumptions stated in Lemma 5 hold. If $g \in C^{2 p+2}(J)$, then

$$
D^{\alpha} A_{h} L^{*} C_{r, h} g(t)=\sum_{i=k}^{p} C_{\alpha, r, i, 1}(t) h^{2 i}+R_{\alpha, r, h, 1}(t), \quad t \in J, 0 \leq \alpha \leq m,
$$

$$
D^{\alpha} B_{h} L^{*} C_{r, h} g(t)=\sum_{i=k}^{p} C_{\alpha, r, i, 2}(t) h^{2 i}+R_{\alpha, r, h, 2}(t), \quad t \in\left\{t_{n}\right\}, 0 \leq \alpha \leq m-1,
$$

where all $C_{\alpha, r, i, j}(t)$ are independent of $h, C_{\alpha, r, i, 1} \in C^{2 p+2}(J)$ and $C_{\alpha, r, i, 2} \in$ $C^{2 p+2-2 i}(J) ; R_{\alpha, r, h, j} \in C(J)$ and satisfy $\left\|R_{\alpha, r, h, j}\right\|_{\infty} \leq C h^{2 p+2}(j=1,2)$.

Proof. Lemma 3 implies that $\left(\pi_{h}-I\right) g$ is "expansible". Let $G(t, s)$ denote the Green's function of the differential operator $L$, then $G \in C^{2 p+2}\left(\Omega_{1}\right) \cap C^{2 p+2}\left(\Omega_{2}\right)$. Thus, the inductive method, together with Lemma 4 , infers that $L^{*} A_{h}$ and $L^{*} B_{h}$ are "expansible". Furthermore, we know that $L^{-1}\left(\pi_{h}-I\right) \sum_{j=1}^{\lambda-1} M_{h}^{j} L^{*} C_{r, h} g$ and $\left(\pi_{h}-I\right)\left(I+\sum_{j=1}^{\lambda} M_{h}^{j}\right) L^{*} C_{r, h} g$ are "expansible". Noting that the operator $L^{-1}\left(I-K L^{-1}\right)^{-1}: C^{2 p+2}(J) \rightarrow C^{2 p+2+m}(J)$ is independent of $h,(3.10)$ implies this lemma.

The following lemma can be verified by [5] Lemma 1-Lemma 4 (refer to the proofs of Lemma 4).

Lemma 7. Under the conditions of Lemma 5, we have

$$
\left\|K L^{-1}\left(\pi_{h}-I\right)\right\|_{C^{2 k}(J) \rightarrow C^{2 k}(J)} \leq C h^{2 k} .
$$

\section{Proofs of the main Results}

Proof of Theorem 1. (i) Using (3.2) we obtain (Lemma 5 implies $L_{1} u \in C^{2 p+2}(J)$ )

$$
\begin{aligned}
\|e\|_{W_{\infty}^{m}} & \leq\left\|\left(L_{1}-\pi_{h} K_{1}\right)^{-1}\right\|_{C(J) \rightarrow C^{m}(J)} \bullet\left\|\left(\pi_{h}-I\right) L_{1} u\right\|_{\infty} \\
& \leq C h^{k} .
\end{aligned}
$$

Thus, for $0 \leq \alpha \leq m$, we have

$$
\begin{aligned}
\left\|D^{\alpha}\left(K_{h} L^{*}\right)^{\mu+1} e\right\|_{\infty} & \leq\left\|\left(K_{h} L^{*}\right)^{\mu+1} e\right\|_{W_{\infty}^{m}} \\
& \leq\left\|K_{h} L^{*}\right\|_{W_{\infty}^{m} \rightarrow W_{\infty}^{m}}^{\mu+1} \bullet\|e\|_{W_{\infty}^{m}} \\
& \leq C h^{\mu+1+k} .
\end{aligned}
$$

If we set $\mu=2 p+1-k$, then

$$
\left\|D^{\alpha}\left(K_{h} L^{*}\right)^{\mu+1} e\right\|_{\infty} \leq C h^{2 p+2}, \quad 0 \leq \alpha \leq m .
$$

On the other hand, $\left(K_{h} L^{*}\right)^{i} K_{h}$ can be written as

$$
\left(K_{h} L^{*}\right)^{i} K_{h}=\left(A_{h} L^{*}+B_{h} L^{*}\right)^{i}\left(A_{h}+B_{h}\right)+K_{h}^{*},
$$

where $K_{h}^{*}$ denotes the term containing the factor

$$
L^{-1} \pi_{h}\left(I-K L^{-1} \pi_{h}\right)^{-1} K L^{-1}\left(\pi_{h}-I\right) M_{h}^{\lambda} .
$$


Let the natural number $\lambda$ be chosen as $\lambda=\left[\frac{p+1}{k}\right]$. Then (by (3.17))

$$
\begin{aligned}
\| L^{-1} & \pi_{h}\left(I-K L^{-1} \pi_{h}\right)^{-1} K L^{-1}\left(\pi_{h}-I\right) M_{h}^{\lambda} \|_{C^{2 k}(J) \rightarrow C^{m}(J)} \\
\leq & \left\|L^{-1} \pi_{h}\right\|_{C(J) \rightarrow C^{m}(J)} \bullet\left\|\left(I-K L^{-1} \pi_{h}\right)^{-1}\right\|_{C(J) \rightarrow C(J)} \\
& \bullet\left\|K L^{-1}\left(\pi_{h}-I\right)\right\|_{C^{2 k}(J) \rightarrow C(J)} \bullet\left\|M_{h}\right\|_{C^{2 k}(J) \rightarrow C^{2 k}(J)}^{\lambda} \\
\leq & C h^{2 k(\lambda+1)}=C h^{2 p+2} .
\end{aligned}
$$

Now we consider $D^{\alpha}\left(A_{h} L^{*}+B_{h} L^{*}\right)^{r}\left(A_{h}+B_{h}\right)$, here $0 \leq \alpha \leq m, 0 \leq r \leq \mu$. Without loss of generality, we assume that $r \geq 1$, thus

$$
D^{\alpha}\left(A_{h} L^{*}+B_{h} L^{*}\right)^{r}\left(A_{h}+B_{h}\right)=D^{\alpha} A_{h} L^{*} C_{r, h}+D^{\alpha} B_{h} L^{*} C_{r, h} .
$$

Since

$$
\begin{aligned}
D^{m} B_{h} L^{*} C_{r, h} & =\left(L^{*}+L\right) B_{h} L^{*} C_{r, h} \\
& =L^{*} B_{h} L^{*} C_{r, h}+\left(\pi_{h}-I\right)\left(I+\sum_{j=1}^{\lambda} M_{h}^{j}\right) L^{*} C_{r, h},
\end{aligned}
$$

and $\bar{\pi}_{h}\left(\pi_{h}-I\right)=0$, thus we have

$$
\bar{\pi}_{h} D^{m} B_{h} L^{*} C_{r, h}=\bar{\pi}_{h} L^{*} B_{h} L^{*} C_{r, h} .
$$

By (3.15), (3.16) and (4.4), this leads to

$$
\begin{gathered}
\bar{\pi}_{h} D^{\alpha}\left(A_{h} L^{*}+B_{h} L^{*}\right)^{r}\left(A_{h}+B_{h}\right) g(t)=\bar{\pi}_{h} D^{\alpha}\left(A_{h} L^{*}+B_{h} L^{*}\right) C_{r, h} g(t) \\
=\sum_{j=k}^{p} h^{2 j} \bar{\pi}_{h} C_{\alpha, r, j}(t)+\bar{\pi}_{h} R_{\alpha, r, h}(t), \quad 0 \leq \alpha \leq m, t \in J,
\end{gathered}
$$

where $0 \leq r \leq \mu ; g \in C^{2 p+2}(J) ; C_{\alpha, r, j}(t)$ are independent of $h$, and $C_{\alpha, r, j} \in$ $C^{2 p+2-2 j}(J) ; R_{\alpha, r, h} \in C(J)$, and $\left\|R_{\alpha, r, h}\right\|_{\infty} \leq C h^{2 p+2}$.

Using (3.1), together with (4.1), (4.2), (4.3) and (4.5), yields that (note that $\left.\left\|\left(\bar{\pi}_{q h}-I\right) C_{\alpha, r, j}\right\|_{\infty} \leq C h^{2 p+2-2 j}\right)$

$$
\bar{\pi}_{h} D^{r} e(t)=\sum_{j=k}^{p} C_{r, j}(t) h^{2 j}+\bar{R}_{r, h}(t),
$$

where $\bar{R}_{r, h} \in C(J)$ satisfies $\left\|\bar{R}_{r, h}\right\|_{\infty} \leq C h^{2 p+2}$.

Noting that

$$
\bar{\pi}_{h} D^{r} u_{h}-D^{r} u=\bar{\pi}_{h} D^{r} e+\left(\bar{\pi}_{h}-I\right) D^{r} u
$$

and

$$
\left\|\left(\bar{\pi}_{h}-I\right) D^{r} u\right\|_{\infty} \leq C h^{2 p+2}
$$

using (4.6), we readily deduce (2.4).

(ii) can be derived in an analogous way. (For this particular case, (3.1) becomes $e=K_{h} L_{1} u$. Moreover, we have $D^{m} B_{h}=\left(\pi_{h}-I\right)\left(I+\sum_{j=1}^{\lambda} M_{h}^{j}\right)$ and $\widetilde{\pi}_{h}\left(\pi_{h}-I\right)=0$. Besides, we need to use an obvious expansion of $M_{h}^{j} L_{1} u$.)

Proof of Theorem 2. (i) Set $T_{h}=\bar{\pi}_{h} D^{m} Q_{h} L_{1}^{-1}-I$. The expansion (2.7) may be written in the form

$$
T_{h} D^{m} u=\sum_{j=k}^{p} h^{2 i} C_{i}+R_{h},
$$


where $p=k(r+1)-1$; the functions $C_{m, i}(t)$ and $R_{m, h}(t)$ are, respectively, abbreviated to $C_{i}$ and $R_{h}$. Thus

$$
T_{h}^{2} D^{m} u=\sum_{i=k}^{p} h^{2 i} T_{h} C_{i}+T_{h} R_{h}
$$

Note that $L_{1}^{-1} C_{i}$ and $Q_{h} L_{1}^{-1} C_{i}$ can be regarded, respectively, as the exact solution and collocation solution of the following auxiliary integro-differential boundary value problem

$$
\left(L_{1}-K_{1}\right) v=f_{i},
$$

with $f_{i}=\left(L_{1}-K_{1}\right) L_{1}^{-1} C_{i}$. Thus, when $u$ and $u_{h}$ are, respectively, replaced by $L_{1}^{-1} C_{i}$ and $Q_{h} L_{1}^{-1} C_{i}$, expansion (2.7) will still be valid.

Let $2 p+2-2 i>2 k$ (i.e. $i<p+1-k$ ). Then

$$
T_{h} C_{i}=\sum_{j=k}^{p-i} h^{2 j} \bar{C}_{i, j}+\bar{R}_{i, h},
$$

where $\bar{C}_{i, j} \in C^{2 p+2-2 i-2 j}(J) ;\left\|\bar{R}_{i, h}\right\|_{\infty} \leq C h^{2(p-i)+2}$.

If $2 p+2-2 i \leq 2 k$ (i.e. $i \geq p+1-k$ ), then we have

$$
\left\|T_{h} C_{i}\right\|_{\infty} \leq C h^{2 p+2-2 i}\left\|C_{i}\right\|_{2 p+2-2 i, \infty} .
$$

Thus, if we substitute (4.8) and (4.9) into (4.7), then

$$
T_{h}^{2} D^{m} u=\sum_{r=2 k}^{p} h^{2 r} C_{r, 1}+R_{h, 1}
$$

where $C_{r, 1}=\sum_{j+i=r} \bar{C}_{i, j} \in C^{2 p+2-2 r}(J) ;\left\|R_{h, 1}\right\|_{\infty} \leq C h^{2 p+2}$.

Successively, we obtain

$$
\left\|T_{h}^{r+1} D^{m} u\right\|_{\infty} \leq C h^{2 p+2}=C h^{2 k(r+1)} .
$$

On the other hand, we have (note that $Q_{h} u=u_{h}$ )

$$
\begin{aligned}
T_{h}^{r+1} D^{m} u & =\left(\bar{\pi}_{h} D^{m} Q_{h} L_{1}^{-1}-I\right)^{r+1} D^{m} u \\
& =\sum_{j=0}^{r}(-1)^{j} C_{r+1}^{j}\left(\bar{\pi}_{h} D^{m} Q_{h} L_{1}^{-1}\right)^{r-j} \bar{\pi}_{h} D^{m} u_{h}-(-1)^{r} D^{m} u \\
& =(-1)^{r} D^{m}\left(u_{h, r}-u\right),
\end{aligned}
$$

and by (4.10), this leads to (2.7).

(ii) (2.8) can be deduced in the same way (using (2.6)).

Remark 4.1. The interpolation correction technique introduced in this paper is also suitable for integro-differential equations with other kinds of boundary conditions. For example, it is fit for integro-differential boundary value problems generated by the regularization method for the first kind Fredholm integral equations (refer to $[9])$.

Remark 4.2. When the integrals appearing in the collocation equation (2.2) cannot be evaluated analytically, the fully discretized form of (2.2) will be obtained by approximating these integrals by product integration techniques. It can be verified by using our method that the corresponding approximation has the same asymptotic properties as $u_{h}$, provided we select the Gauss-type quadrature weights. 


\section{NUMERICAL EXAMPLES}

To illustrate the theoretical results stated in Section 2 and compare them with the corresponding results given in [10], we consider the examples:

\section{Example 1.}

$$
\left\{\begin{array}{l}
u^{\prime \prime}(t)-p(t) u(t)+\frac{2 \pi^{2}}{9} \int_{0}^{1} \cos \frac{\pi}{3}(t-s) u(s) d s=f(t), \quad t \in[0,1], \\
u(0)-2 u(1)=0, \quad u^{\prime}(0)=0,
\end{array}\right.
$$

with $f(t)$ chosen so that $u(t)=\cos \frac{\pi}{3} t\left(p(t) \equiv 0, f(t)=\frac{\sqrt{3} \pi}{6} \cos \frac{\pi}{3}(1-t)\right.$ or $p(t) \equiv$ $\frac{\sqrt{3} \pi}{6}, f(t)=\frac{\sqrt{3} \pi}{6} \sin \frac{\pi}{6}(1-2 t)$.

The numerical results are obtained with $k=1\left(c_{1}=0, c_{2}=1, c_{1}^{\prime}=\frac{1}{2}\right)$ and with $k=2\left(c_{1}=0, c_{2}=\frac{1}{2}, c_{3}=1, c_{1}^{\prime}=\frac{3-\sqrt{3}}{6}, c_{2}^{\prime}=\frac{3+\sqrt{3}}{6}\right)$. The multilevel correction estimate (2.8) is confirmed by Table 1, and (2.7) is confirmed by Table 2 .

\section{Example 2.}

$$
u(t)-2 \int_{0}^{1} \cos \frac{\pi}{3}(t-s) u(s) d s=f(t), \quad t \in[0,1],
$$

with $f(t)$ chosen as $f(t)=-\frac{3 \sqrt{3}}{2 \pi} \cos \frac{\pi}{3} t$ so that $u(t)=\cos \frac{\pi}{3} t$.

Let $u_{h} \in S_{k-1, h}^{(-1)}$ be the Galerkin approximation to this equation, and $\widetilde{u}_{h, r}$ denote the corresponding $r$ th-level iterated corrected approximations (see [10]). The error estimates are given in Table 3.

The numerical results confirm our inference.

\begin{tabular}{|c|c|}
\hline$k=1$ & $k=2$ \\
\hline $\bar{N}\|\| \bar{u}_{h, 2}-u \|_{\infty} \mid$ rates & $\bar{N}\|\| \bar{u}_{h, 1}-u \|_{\infty} \mid$ rates \\
\hline $10|2.58 \mathrm{D}-6|$ & \\
\hline \begin{tabular}{l|l|l}
20 & $4.18 \mathrm{D}-8$ & 5.96
\end{tabular} & $15|3.27 \mathrm{D}-8|$ \\
\hline \begin{tabular}{l|l|l|}
40 & $6.91 \mathrm{D}-10$ & 5.93
\end{tabular} & \begin{tabular}{l|l|l|}
30 & $1.33 \mathrm{D}-10$ & 7.93 \\
\end{tabular} \\
\hline
\end{tabular}

TABLE 1

\begin{tabular}{|c|c|}
\hline$k=1$ & $k=2$ \\
\hline$\overline{\mid N\left\|u_{h, 2}-u\right\|_{\infty}}$ rates & $\overline{\left|N\left\|u_{h, 1}-u\right\|_{\infty}\right| \text { rates }}$ \\
\hline $10|3.01 \mathrm{D}-6|$ & \\
\hline \begin{tabular}{l|l|l|}
20 & $4.87 \mathrm{D}-8$ & 5.95
\end{tabular} & $15|4.34 \mathrm{D}-8|$ \\
\hline \begin{tabular}{l|l|l}
40 & $7.94 \mathrm{D}-10$ & 5.91
\end{tabular} & \begin{tabular}{l|l|l|}
$30.77 \mathrm{D}-10$ & 7.92
\end{tabular} \\
\hline
\end{tabular}

TABLE 2

\begin{tabular}{|c|c|}
\hline$k=1$ & $k=2$ \\
\hline 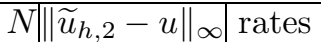 & $\overline{|N|\left\|\widetilde{u}_{h, 1}-u\right\|_{\infty} \mid \text { rates }}$ \\
\hline $10|2.74 \mathrm{D}-6|$ & \\
\hline \begin{tabular}{|c|c|c|}
20 & $4.53 \mathrm{D}-8$ & 5.94
\end{tabular} & $15|3.81 \mathrm{D}-8|$ \\
\hline \begin{tabular}{l|l|l|}
40 & $7.38 \mathrm{D}-10$ & 5.92
\end{tabular} & \begin{tabular}{l|l|l|}
30 & $1.64 \mathrm{D}-10$ & 7.91
\end{tabular} \\
\hline
\end{tabular}

TABLE 3 


\section{ACKNOWLEDGEMENT}

The author is grateful to the referee for his constructive criticism which helped to improve the presentation of the paper.

\section{REFERENCES}

[1] C. de Boor and B. Swartz, Collocation at Gauss points, SIAM J. Numer. Anal. 10(1973), 582-606. MR 51:9528

[2] J. Lei and X. Huang, The projection methods for operator equations, Wuhan University Press 1987.

[3] L. M. Delves and J. L. Mohamed, Computational methods for integral equations, Cambridge University Press 1985. MR 87j:65159

[4] Q. Hu, Extrapolation of finite element solutions to a class of integrodifferential equations, Natur. Scie. J. Xiangtan Univ. 14(1992), 28-34. MR 93g:65103

[5] Q. Hu, Extrapolation for collocation solutions of Volterra integro-differential equations, Chinese J. Numer. Math. Appl. 18(1996), No.2, 28-37. CMP 97:08

[6] Q. Hu, Acceleration of Convergence for Galerkin method solutions to Fredholm Integrodifferential Equations, Syst. Sci. and Math. 17(1997), 14-18. CMP 97:14

[7] W. Volk, The numerical solution of linear integro-differential equations by projection methods, J. Integ. Equations 9. Suppl.(1985), 171-190. MR 87g:65168

[8] W. Volk, The iterated Galerkin methods for linear integrodifferential equations, J Comp. Appl. Math. 21(1988), 63-74. MR 89a:65201

[9] A. Zhou, An extrapolation method for finite element approximation of integro-differential equations with parameters, Syst. Sci. and Math. 3(1990), 278-285. MR 93h:65173

[10] Q. Zhu and L. Cao, Multilevel correction for FEM and BEM, Natur. Scie. J. Xiangtan Univ. 14(1992), 1-5. MR 93g:65105

Institute of Mathematics, Chinese Academy of Science, Beijing 100080, China 